\title{
REVERBERATION MAPPING ANALYSIS OF THE BROAD- LINE REGION IN SEYFERT GALAXY NGC 4151
}

\author{
S.J. XUE \\ The Institute for Physical and Chemical Research (RIKEN) \\ Hirosawa 2-1, Wako, Saitama 351-01, Japan \\ Shaanxi Astronomical Observatory, Academia Sinica \\ Lintong 710600, China \\ AND \\ F.Z. CHENG \\ The University of Science $\&$ Technology of China (USTC) \\ Heifei 230026, China
}

\section{Introduction}

One of the primary goals of AGN variability studies has been to determine the size of broad-line region (BLR) through the reverberation mapping technique. In a recent international multiwavelength spectroscopic monitoring campaign, NGC 4151 has been observed intensively by ground-based telescopes for a period of over 2 months, with a typical temporal resolution of 1 day. The main result from this optical campaign is that finding the variation in the emission line flux $(H \beta$ or $H \alpha)$ lagging the continuum by 0-3 days (1993 campaign: Kaspi et al. 1996). This is in contrast to the past results in which a time lag of $9 \pm 2$ days was found for the same emission line (1988 campaign: Maoz et al. 1991). Such a BLR "size problem" may be caused by a different variability timescale of the ionizing continuum or a real change in BLR gas distribution in the $5.5 \mathrm{yr}$ interval between the two watch campaigns. In order to clarify which of the two possibilities is most likely the real case, we performed further reverberation analysis on both optical datasets. 


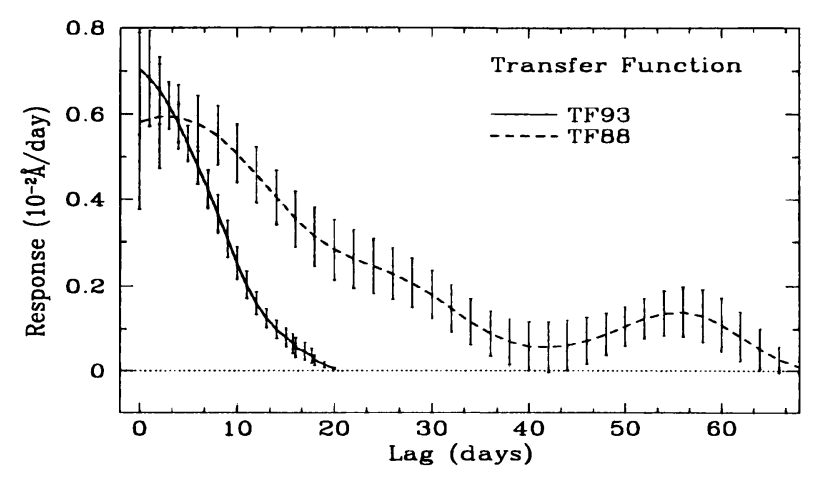

Figure 1. Transfer functions recovered from two optical watch campaigns.

\section{Monte-Carlo Simulation}

We first evaluated the uncertainties associated with the lag measurement caused by any "ionizing" continuum behaviors via Monte-Carlo simulations. The results show that, for the 1993's continuum behavior, if the BLR structure keeps stationary as it was in 1988 , we would get the BLR size of $10.5 \pm 6.3$ days in the $95 \%$ error range. This is in sharp contrast to the practical result of $0-3$ days. We thus concluded that the hypothesis of the stationary BLR structure between the $5.5 \mathrm{yr}$ interval of the two observation campaigns is highly excluded.

\section{Comparison of Transfer Functions}

We then worked out BLR transfer functions TF93 and TF88 for both datasets using the Regularized Linear Inversion method (Krolik \& Done, 1995). Figure 1 shows the significant difference between the shapes of the two. In contrast to TF88, TF93 reveals a much "condense" BLR, which is most likely a shell with 10 day thickness and the inner radius of $\lesssim 1$ day. The dramatic change of the transfer function indicates the real difference in BLR gas distribution between 1988 and 1993. This observed BLR evolution not only naturally explains the present "size problem", but also provides an exciting new dimension in AGN variability studies.

\section{References}

Kaspi, S., et al. 1996, ApJ, 470, 336

Krolik, J.H. \& Done, C. 1995, ApJ, 440, 166

Maoz, D., Netzer, H., Leibowitz, E. et al., 1991, ApJ, 421, 34 